\title{
Improved Parabolization of the Euler Equations
}

\author{
Aaron Towne* and Tim Colonius ${ }^{\dagger}$ \\ California Institute of Technology, Pasadena, CA, 91106, USA
}

\begin{abstract}
We present a new method for stability and modal analysis of shear flows and their acoustic radiation. The Euler equations are modified and solved as a spatial initial value problem in which initial perturbations are specified at the flow inlet and propagated downstream by integration of the equations. The modified equations, which we call one-way Euler equations, differ from the usual Euler equations in that they do not support upstream acoustic waves. It is necessary to remove these modes from the Euler operator because, if retained, they cause instability in the spatial marching procedure. These modes are removed using a two-step process. First, the upstream modes are partially decoupled from the downstream modes using a linear similarity transformation. Second, the error in the first step is eliminated using a convergent recursive filtering technique. A previous spatial marching method called the parabolized stability equations uses numerical damping to stabilize the march, but this has the unintended consequence of heavily damping the downstream acoustic waves. Therefore, the one-way Euler equation could be used to obtain improved accuracy over the parabolized stability equations as a low-order model for noise simulation of mixing layers and jets.
\end{abstract}

\section{Introduction}

The high cost of direct and large-eddy simulation of jet noise has prompted the development of reducedorder methods based on instability waves. Experiments have long suggested that the breakdown of coherent structures at the end of the potential core is the dominant source of sound at small angles to the jet axis, where sound is most intense. ${ }^{1-4}$ These coherent structures can be quantitatively identified as instability waves in both forced ${ }^{5}$ and natural ${ }^{6}$ jets. Therefore, accurate modeling of instability waves has the potential to provide an accurate, low-cost jet noise model.

The most inexpensive instability wave-based approaches are locally parallel methods based on linear stability theory. ${ }^{7,8}$ These methods have provided insight into the role of the Kelvin-Helmholtz instability in jet noise production and the sensitivity of this instability to various flow parameters. However, the locally parallel assumption of these methods limit their ability to accurately capture nonparallel effects and the downstream evolution of the instability waves.

To fully account for nonparallel effects, global mode analysis has been used ${ }^{9}$ to capture the spatial growth and decay of near-field instability waves in jets and their associated acoustic radiation. The trade-off is that global modes are expensive to compute, limiting their utility as a reduced-order model.

In between these two extremes lies an ad hoc generalization of linear stability theory call the parabolized stability equations (PSE). ${ }^{10}$ PSE can partially capture nonparallel and nonlinear effects, and has been shown to accurately model many convectively unstable flows. In particular, our group has previously shown that linear PSE can produce quantitatively accurate near-field ${ }^{11}$ and reasonable far-field ${ }^{12}$ sound predictions for unforced turbulent jets at a fraction of the cost of LES or global mode analysis.

The efficiency of PSE is achieved by using a spatial marching technique in the streamwise direction. Initial conditions are specified at the jet inlet and propagated downstream by integration of the PSE equations. Because of this one-way solution strategy, upstream propagating waves cannot be captured by PSE. This is a minor loss, however, because of the substantial downstream directivity of jet noise.

Care must be taken to assure a stable downstream march. This is the case because, despite their name, the parabolized stability equations are not, in fact, parabolic. Even though PSE cannot capture upstream

*Ph.D. student.

${ }^{\dagger}$ Professor, AIAA Associate Fellow. 
sound, the PSE operator still contains upstream acoustic modes which constitute an elliptic effect in the frequency domain. These elliptic modes are a consequence of the inherent boundary value nature of the subsonic Euler equations. Specifically, decaying upstream evanescent modes are wrongly interpreted by the PSE operator as growing downstream modes, causing exponential instability in the march if they are not eliminated. Eliminating these elliptic modes is the goal of parabolization. The traditional PSE method accomplishes this by using an implicit Euler axial discretization along with a restriction on the minimum step size. This has the effect of numerically damping the upstream acoustic modes such that they move inside the unit circle in the discrete spectral space. If a step size below the restriction is used, these modes again move outside of the unit circle and cause unbounded instability. ${ }^{13}$

An unintended consequence of the numerical damping used to eliminate the upstream acoustic modes is that the desired downstream acoustic modes are also heavily damped. Additionally, the minimum step size restriction in some cases prevents adequate convergence of the solution. Several alternative methods ${ }^{14,15}$ have been devised to remove the upstream acoustic mode from the PSE operator and reduce the minimum allowable step size, but each of these methods still has catastrophic effects on the downstream acoustic radiation.

In this paper, we introduce a new spatial marching technique that can be used to model instability waves and their associated acoustic radiation. Our method explicitly removes the upstream acoustic modes from the linearized Euler equations while accurately retaining the downstream acoustic modes. The resulting equations can be integrated without the use of numerical damping, allowing the downstream acoustic field to be accurately modeled. Our method draws from techniques for generating nonreflecting boundary conditions. Guddati $^{16}$ developed a one-way marching technique for the elastic wave equation based on a different class of nonreflecting boundary conditions, but it has not, to our knowledge, been extended to equations for fluid dynamics.

\section{Method}

In this section, we outline our method for parabolizing the Euler equations. We first discuss relevant aspects of the nature of the subsonic Euler equations, then present the two steps of our method, and finally consider computational cost. We will focus on the two-dimensional Euler equations for ease of presentation, but our method can be directly applied to three dimensional Cartesian and cylindrical coordinates as well, and can be extended to the Navier-Stokes equations.

\section{II.A. The linearized Euler equations}

We begin with the two-dimensional compressible Euler equations:

$$
\begin{gathered}
\frac{\partial \nu}{\partial t}+u_{x} \frac{\partial \nu}{\partial x}+u_{y} \frac{\partial \nu}{\partial y}-\nu\left(\frac{\partial u_{x}}{\partial x}+\frac{\partial u_{y}}{\partial y}\right)=0, \\
\frac{\partial u_{x}}{\partial t}+u_{x} \frac{\partial u_{x}}{\partial x}+u_{y} \frac{\partial u_{x}}{\partial y}+\nu \frac{\partial p}{\partial x}=0, \\
\frac{\partial u_{y}}{\partial t}+u_{x} \frac{\partial u_{y}}{\partial x}+u_{y} \frac{\partial u_{y}}{\partial y}+\nu \frac{\partial p}{\partial y}=0, \\
\frac{\partial p}{\partial t}+u_{x} \frac{\partial p}{\partial x}+u_{y} \frac{\partial p}{\partial y}+\gamma p\left(\frac{\partial u_{x}}{\partial x}+\frac{\partial u_{y}}{\partial y}\right)=0 .
\end{gathered}
$$

The fluid is a perfect gas with specific heat ratio $\gamma$. We have chosen as independent variables specific volume, axial velocity, transverse velocity, and pressure. All variables have been appropriately nondimensionalized by an ambient sound speed and density and a problem dependent length-scale. We decompose the flow-field $\boldsymbol{q}=\left\{\nu, u_{x}, u_{y}, p\right\}^{T}$ into a steady baseflow and fluctuations about this baseflow:

$$
\boldsymbol{q}(x, y, t)=\overline{\boldsymbol{q}}(x, y)+\boldsymbol{q}^{\prime}(x, y, t) .
$$

If non-linear products of fluctuations are neglected and the baseflow satisfies equation (1), then the fluctuations satisfy the linearized, two-dimensional, compressible Euler equations: 


$$
\frac{\partial \boldsymbol{q}^{\prime}}{\partial t}+A(\overline{\boldsymbol{q}}) \frac{\partial \boldsymbol{q}^{\prime}}{\partial x}+B(\overline{\boldsymbol{q}}) \frac{\partial \boldsymbol{q}^{\prime}}{\partial y}+L(\overline{\boldsymbol{q}}) \boldsymbol{q}^{\prime}=0
$$

with

$$
\begin{gathered}
A=\left[\begin{array}{cccc}
\bar{u}_{x} & -\bar{\nu} & 0 & 0 \\
0 & \bar{u}_{x} & 0 & \bar{\nu} \\
0 & 0 & \bar{u}_{x} & 0 \\
0 & \gamma \bar{p} & 0 & \bar{u}_{x}
\end{array}\right], \quad B=\left[\begin{array}{cccc}
\bar{u}_{y} & 0 & -\bar{\nu} & 0 \\
0 & \bar{u}_{y} & 0 & 0 \\
0 & 0 & \bar{u}_{y} & \bar{\nu} \\
0 & 0 & \gamma \bar{p} & \bar{u}_{y}
\end{array}\right], \\
L=\left[\begin{array}{ccccc}
-\left(\frac{\partial \bar{u}_{x}}{\partial x}+\frac{\partial \bar{u}_{y}}{\partial y}\right) & \frac{\partial \bar{\nu}}{\partial x} & \frac{\partial \bar{\nu}}{\partial y} & 0 & \\
\frac{\partial \bar{p}}{\partial x} & \frac{\partial \bar{u}_{x}}{\partial x} & \frac{\partial \bar{u}_{x}}{\partial y} & 0 & \\
\frac{\partial \bar{p}}{\partial y} & \frac{\partial \bar{u}_{y}}{\partial x} & \frac{\partial \bar{u}_{y}}{\partial y} & 0 & \\
0 & \frac{\partial \bar{p}}{\partial x} & \frac{\partial \bar{p}}{\partial y} & \gamma\left(\frac{\partial \bar{u}_{x}}{\partial x}+\frac{\partial \bar{u}_{y}}{\partial y}\right)
\end{array}\right]
\end{gathered}
$$

Next, the fluctuations are further decomposed into a summation of frequency modes:

$$
\boldsymbol{q}^{\prime}(x, y, t)=\sum_{n} \hat{\boldsymbol{q}}_{n}(x, y) e^{-\imath \omega_{n} t} .
$$

In choosing $\omega_{n}$ to be real-valued, we restrict our analysis to flows that are stationary in time. Inserting this decomposition into equation (3), we have for each frequency mode

$$
-\imath \omega_{n} \hat{\boldsymbol{q}}_{n}+A(\overline{\boldsymbol{q}}) \frac{\partial \hat{\boldsymbol{q}}_{n}}{\partial x}+B(\overline{\boldsymbol{q}}) \frac{\partial \hat{\boldsymbol{q}}_{n}}{\partial y}+L(\overline{\boldsymbol{q}}) \hat{\boldsymbol{q}}_{n}=0 .
$$

Since we wish to interpret this equations as a spatial evolution equation in the streamwise $x$ direction, we rewrite equation (6) as

$$
\frac{\partial \hat{\boldsymbol{q}}_{n}}{\partial x}=-A^{-1}\left(-\imath \omega_{n} I+B D_{y}+L\right) \hat{\boldsymbol{q}}_{n}:=M_{n} \hat{\boldsymbol{q}}_{n} .
$$

The inverse of the matrix $A$ exists as long as $0<\bar{u}_{x}<1$, so the baseflow in the axial direction must be downstream and subsonic everywhere. The operator $D_{y}$ represents the transverse derivative, and will be approximated by a differentiation matrix upon discretization of the equations. The streamwise evolution of equation (7) is governed by the eigenvalues and eigenvectors of the operator $M_{n}$. Analytical solutions for these quantities do not exist for a general spatially inhomogeneous baseflow.

To understand the structure of $M_{n}$, it is helpful to look at the special case of a uniform baseflow, for which analytical expressions can be derived by taking a Fourier transform in $y$. Then, the eigenvalues and left eigenvectors are

$$
\begin{gathered}
\lambda_{s}=\frac{k}{M_{x}}, \\
\lambda_{\omega}=\frac{k}{M_{x}}, \\
\lambda_{ \pm}(z)=k \frac{-M_{x} \pm \mu(z)}{1-M_{x}^{2}}, \\
U(z)=\left[\begin{array}{c}
\boldsymbol{u}_{s}^{T} \\
\boldsymbol{u}_{\omega}^{T} \\
\boldsymbol{u}_{+}^{T} \\
\boldsymbol{u}_{-}^{T}
\end{array}\right]=\left[\begin{array}{cccc}
1 & 0 & 0 & d^{2} \\
0 & -\bar{M}_{x} z & 1 & -d z \\
0 & 1 & \bar{M}_{x} z & d \mu(z) \\
0 & 1 & \bar{M}_{x} z & -d \mu(z)
\end{array}\right] .
\end{gathered}
$$

Here, $M_{x}=\bar{u}_{x} / \bar{c}$ is the Mach number, $d=\bar{\nu} / \bar{c}$ is the acoustic admittance, $k=\omega / \bar{c}$ is the reduced frequency, $\bar{c}$ is the local sound speed of the baseflow, $\eta$ is the transverse Fourier wavenumber, $z=\eta / k$ is a scaled transverse wavenumber, and the function $\mu(z)$ is given by 


$$
\mu(z)=\sqrt{1-\left(1-M_{x}^{2}\right) z^{2}} .
$$

The first two eigenvalues, $\lambda_{s}$ and $\lambda_{\omega}$, describe the evolution of entropy and vorticity, respectively. These modes convect downstream with group velocity $\bar{u}_{x}$. The eigenvalues $\lambda_{ \pm}$represent acoustic branches of the spectrum, consisting of acoustic waves propagating in all directions as well as evanescent waves which decay exponentially in $y$. It can be easily verified ${ }^{17}$ that for all $z, \lambda_{ \pm}$have positive and negative group velocities, respectively. These eigenvalues are shown graphically in figure 1. The parts of the acoustic branches that lie along the stability boundary are the propagating acoustic waves and the vertical parts are the evanescent modes. Although the preceding analysis is strictly valid only for a uniform baseflow, inhomogeneous flows like jets and mixing layers contain modes that can be grouped into families with the same qualitative properties as the four uniform flow modes.

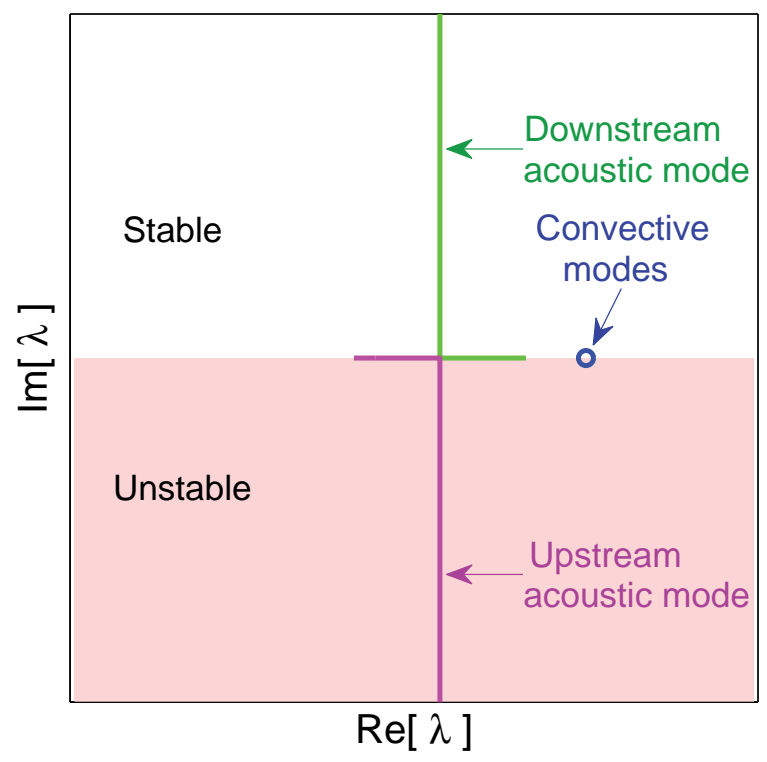

Figure 1: Uniform flow Euler spectrum.

Overall, the linearized Euler equations support three downstream propagating modes $\left(\lambda_{s}, \lambda_{\omega}\right.$, and $\left.\lambda_{+}\right)$ and one upstream mode $\left(\lambda_{-}\right)$. This result is reflective of the inherent boundary value nature of the subsonic Euler equations. The downstream modes should be specified at the domain inlet and the upstream mode should be specified at the domain outlet.

If instead the problem is solved as in initial value problem in space - by specifying all modes at the inlet and marching the solution downstream - the upstream acoustic mode will cause instability in the march. Mathematically, this instability occurs because $\lambda_{-}$takes on complex values with negative imaginary part (see figure 1), causing exponential growth of the mode. As mentioned in the introduction, PSE achieves a stable downstream march by numerically damping the upstream mode. This effectively eliminates the instability, but also heavily damps the downstream acoustic modes. Instead, we will develop a method which completely removes the upstream acoustic modes from the Euler equations while retaining all of the downstream modes. This will result in a new one-way Euler operator that accurately models the downstream Euler modes, but unlike the full Euler operator, can be stably integrated in the downstream direction.

\section{II.B. Partial decoupling of dynamics}

Our method contains two steps. First, the upstream and downstream dynamics contained within the Euler equations are partially decoupled. In what follows, we drop the subscript $n$ which indicated the frequency mode for notational brevity. Each mode is treated independently in the same manner. Suppose that there exists a linear similarity transformation $T_{\text {diag }}$ such that the change of variables 


$$
\boldsymbol{\psi}=\left\{\begin{array}{c}
\boldsymbol{\psi}_{+} \\
\boldsymbol{\psi}_{-}
\end{array}\right\}=T_{\text {diag }} \hat{\boldsymbol{q}}
$$

upon substitution into equation (7), results in a block diagonalized system of equations

$$
\frac{d}{d x}\left\{\begin{array}{l}
\boldsymbol{\psi}_{+} \\
\boldsymbol{\psi}_{-}
\end{array}\right\}=\left[\begin{array}{cc}
D_{++} & 0 \\
0 & D_{--}
\end{array}\right]\left\{\begin{array}{l}
\boldsymbol{\psi}_{+} \\
\boldsymbol{\psi}_{-}
\end{array}\right\},
$$

where all eigenvalues of $D_{++}$have positive group velocity and all eigenvalues of $D_{--}$have negative group velocity. Then, $\boldsymbol{\psi}_{+}$contains all of the possible downstream modes and no upstream modes, while $\boldsymbol{\psi}_{-}$contains all of the upstream modes and no downstream modes. The one-way evolution of this system is specified exactly by the pair of equations

$$
\begin{aligned}
\frac{d}{d x} \boldsymbol{\psi}_{+} & =D_{++} \boldsymbol{\psi}_{+}, \\
\boldsymbol{\psi}_{-} & =0 .
\end{aligned}
$$

In general, the transformation required to block diagonalize the Euler equations is unknown analytically and very expensive to compute numerically. Suppose, instead, that we choose a different transformation $T$ that does not diagonalize the system, but that we expect to be a reasonable approximation of the diagonalizing transformation $T_{\text {diag }}$. Then inserting the change of variables

$$
\phi=\left\{\begin{array}{l}
\phi_{+} \\
\phi_{-}
\end{array}\right\}=T \hat{\boldsymbol{q}}
$$

into equation (7) will give an equation of the form

$$
\frac{d \phi}{d x}=P \phi
$$

with

$$
P=\left[\begin{array}{ll}
P_{++} & P_{+-} \\
P_{-+} & P_{--}
\end{array}\right] .
$$

The off-diagonal terms $P_{+-}$and $P_{-+}$are non-zero and nothing is implied about the eigenstructure of the diagonal matrices $P_{++}$and $P_{--}$. Now, $\phi_{+}$will consist mostly of downstream modes, but will also contain some upstream content. Likewise, $\phi_{-}$will be primarily made up of upstream modes, but will also contain some downstream content. In other words, the transformation has partially decoupled the upstream and downstream dynamics.

The uniform flow Euler eigenvectors given by equation (9) are a useful guide for choosing a transformation for the Euler equations. If in fact we are working with a truly uniform flow, then the choice $T=F_{y}^{-1}\{U(z)\}$, with the function $\mu(z)$ replaced by a convergent rational approximation of the square root, will completely diagonalize the system. The operator $F_{y}^{-1}$ represents the inverse transverse Fourier transform. This is the approach used in developing one-way approximations of the wave equations. ${ }^{18,19}$ For a non-uniform flow, in which case the exact diagonalizing transformation is not known, the choice $T=F_{y}^{-1}\{U(z)\}$ still gives a good, although incomplete, decoupling. The downside of this choice is that the inverse Fourier transform of the rational approximation of the non-local function $\mu(z)$ results in a $P$ which is a full matrix upon discretization in $y$. This turns out to be very undesirable for the second step of our method.

Instead, we use the even simpler transformation $T=U(z=0)$. Under this transformation, $\phi$ becomes the well-known one-dimensional characteristic variables:

$$
\phi=\left\{\begin{array}{c}
\hat{s} \\
\hat{u}_{y} \\
\hat{r} \\
\hat{l}
\end{array}\right\}=\left[\begin{array}{cccc}
1 & 0 & 0 & d^{2} \\
0 & 0 & 1 & 0 \\
0 & 1 & 0 & d \\
0 & 1 & 0 & -d
\end{array}\right]\left\{\begin{array}{c}
\hat{\nu} \\
\hat{u}_{x} \\
\hat{u}_{y} \\
\hat{p}
\end{array}\right\} .
$$

The characteristic variables are an entropy-like variable $\hat{s}$ and partially decoupled right and left acousticlike variables $\hat{r}$ and $\hat{l}$. The first three make up the approximate upstream variable $\phi_{+}$and the last one 
is the approximate downstream variable $\phi_{-}$. Their name comes from the fact that this transformation completely decouples the upstream and downstream modes for the one-dimensional Euler equations. In a two dimensional setting, the transformation will perfectly decouple waves traveling directly up or downstream, and the quality will decrease for waves traveling at increasing angels from the axis. This choice maintains the sparsity of the chosen transverse discretization.

Since the upstream and downstream dynamics are now partially decoupled, a possible approach would be to ignore the remaining coupling between the upstream and downstream dynamics and set $\phi_{-}=0$. The nature of the error caused by this approximation is revealed if we examine the relationship between the true upstream and downstream variables and the approximate ones. Since both the (hypothetical) diagonalizing transformation $T_{\text {diag }}$ and the approximate diagonalization $T$ are linear similarity transformations, there exists a linear relationship between the fully decoupled and partially decoupled variables:

$$
\left\{\begin{array}{c}
\boldsymbol{\psi}_{+} \\
\boldsymbol{\psi}_{-}
\end{array}\right\}=\left[\begin{array}{ll}
U_{++} & U_{+-} \\
U_{-+} & U_{--}
\end{array}\right]\left\{\begin{array}{l}
\phi_{+} \\
\phi_{-}
\end{array}\right\} .
$$

We make two important observations about equation (18). First, if $\phi_{-}$is set to zero, the true upstream and downstream variables will be incorrect. In particular, certain upstream solution will be allowed since $\boldsymbol{\psi}_{-} \neq 0$ and certain downstream solutions will be disallowed since the $U_{+-} \boldsymbol{\phi}_{-}$contribution to $\boldsymbol{\psi}_{+}$is ignored. Second, notice that there exists a relationship between the approximate upstream and downstream variables $\phi_{+}$and $\phi_{-}$that results in the desired outcome $\boldsymbol{\psi}_{-}=0$. Precisely, this is accomplished when

$$
\phi_{-}=-U_{--}^{-1} U_{-+} \phi_{+}:=F \phi_{+} .
$$

When this condition is met, $\phi$ contains only downstream content. Substituting this result into equation (15) gives:

$$
\begin{gathered}
\frac{d}{d x} \phi_{+}=\left(P_{++}+P_{+-} F\right) \boldsymbol{\phi}_{+}, \\
\boldsymbol{\phi}_{-}=F \boldsymbol{\phi}+.
\end{gathered}
$$

This result is an exact parabolization of the equations if $F$ is known exactly. Our approximate one-way equation is produced by finding an approximate $F$.

\section{II.C. Recursive filtering}

To this end, we introduce a set of auxiliary variables $\left\{\phi^{j}: j=0, \ldots, N_{F}\right\}$ that satisfy the recursion relation

$$
\left(P-b_{j} I\right) \phi^{j}=\left(P-\bar{b}_{j} I\right) \phi^{j+1}, \quad j=0, \ldots, N_{F}-1
$$

with the two-sided termination condition

$$
\begin{aligned}
& \phi_{+}^{0}=\phi_{+} \\
& \phi_{-}^{N_{F}}=0 .
\end{aligned}
$$

Similar recursions were introduced by Givoli \& $\mathrm{Neta}^{20}$ and Hagstrom \& Warburton ${ }^{21}$ within the context of radiation boundary conditions. In that application, the goal is to specify a boundary condition that is transparent to outgoing waves and does not generate spurious incoming waves. This recursion accomplish this by generating an operator at the boundary that is satisfied by waves with outgoing group velocities but not satisfied by waves with incoming group velocities. This is very similar to our goal. We wish to develop an operator that only admits downstream traveling solutions. Our use of these recursions differs only in that we use them to find the proper relationship between $\phi_{+}$and $\phi_{-}$, while boundary condition applications use them to relate time derivatives of these quantities. Additional details about this recursive method within the context of boundary conditions can be found in the literature. ${ }^{20-24}$

The recursion is completely defined by its order $N_{F}$ and the sets of complex integers $\left\{b_{j}, \bar{b}_{j}: j=\right.$ $\left.0, \ldots, N_{F}-1\right\}$, which we call filtering parameters. The system of equations defined by equation (21), equation (22), and equation (23) can be solved for $\phi_{0}^{-}$as a function of $\boldsymbol{\phi}^{+}$. If the filtering parameters are properly selected, the recursions converge to give the correct relationship between these variables as defined by equation (19). To achieve convergence, the $b_{j}$ 's and $\bar{b}_{j}$ 's must be chosen such that they are located near 
the modes we wish to retain and remove, respectively. For the Euler equations, we choose these locations based on our knowledge of the uniform flow eigenvalues:

$$
\begin{gathered}
b_{j} \in\left\{\imath \lambda_{s}, \imath \lambda_{\text {omega }}, \imath \lambda_{+}\right\} \text {for } z \in(0, \infty), \\
\bar{b}_{j} \in \imath \lambda_{-} \text {for } z \in(0, \infty) .
\end{gathered}
$$

To account for baseflow non-uniformity, in addition to varying $z$, we also evaluate the uniform flow eigenvalues using the range of $k$ and $M_{x}$ that exist in the baseflow at the current $x$ location in the march. This ensures that the total set of filtering parameters will contain entries that target all of the actual nonuniform modes.

We will show in the next section that selecting the filtering parameters based on equation (24) is sufficient to achieve convergence. However, we are doubtful that this choice is optimal. Optimal parameters have been derived for the wave equation within the context of nonreflecting boundary conditions that guarantee spectral convergence. ${ }^{22}$ These values are fundamentally linked to a time-domain formulation and cannot be easily translated to the frequency domain. Our selection strategy is an ad hoc frequency domain adaption of these parameters, though we do typically observe spectral convergence. However, it is not clear that optimal time domain parameters are optimal in the frequency domain, and we have reason to expect that they are not. Finding optimal frequency domain parameters is an ongoing aspect of our work.

\section{II.D. Computational Cost}

Implementation of the recursion equations requires the solution of a $4 N_{y} N_{F} \times 4 N_{y} N_{F}$ system of equations, where $N_{y}$ is the number of discretization points in $y$ and $N_{F}$ is the order of the recursion. The number four appears because there are four dependent variables in the two-dimensional Euler equations. We use the software package MUMPS, which is a sparse, multi-frontal, direct solver.

We have found that the variables in the system of equations can be reordered to create a matrix whose bandwidth scales linearly with $N_{F}$ and does not depend on $N_{y}$. Therefore, we expect solving the matrix system to require $\mathcal{O}\left(N_{y} N_{F}^{2}\right)$ operations. However, we have observed that the actual cost scales like $\mathcal{O}\left(N_{y} N_{F}\right)$. In comparison, performing one PSE step requires $\mathcal{O}\left(N_{y} N_{i t}\right)$ operations, where $N_{i t}$ is the number of iterations required to satisfy a nonlinear constraint that is part of the PSE formulation.

\section{Results}

In this section, we present results from two test problems that demonstrate the convergence and utility of our one-way Euler method. First, we model the propagation of acoustic waves in a uniform flow created by a monopole located upstream of the computational domain. Second, we simulate the generation and propagation of acoustic waves in a convectively unstable turbulent mixing layer. For both problems, the one-way Euler results are compared to corresponding PSE solutions.

For all simulations, the equations are discretized in the transverse direction using fourth-order central finite differences and the computational domain is truncated using the super-grid damping layer method ${ }^{25,26}$ to enforce radiation boundary conditions. For the one-way Euler equations, we performed the downstream march using fourth-order Runge-Kutta integration, while PSE uses implicit Euler integration by definition.

\section{III.A. Monopole forcing}

In this problem, a monopole disturbance is placed just upstream of the computational domain in a Mach 0.5 uniform stream. The monopole generates an acoustic field for which an analytical solution exists. Along the inlet of the computational domain, the exact solution is supplied to the one-way Euler equations and to the PSE algorithm as an initial condition. Then, this solution is propagated downstream by the spatial marching routine of each method. Figure 2(a) shows the real part of the pressure field created by the monopole. The wavefronts are not concentric because of the non-zero baseflow moving from left to right. The initial condition is specified at the location of the dashed line, and the solution to the right of this is reproduced by the marching procedures.

The error of the overall full-field solutions produced by PSE and the one-way Euler equations are calculated by comparison to the full-field exact analytical solution. Figure 2(b) contains the error results. The convergence of the one-way Euler solution is roughly spectral as the order of the recursion increases until the 
error levels of at $N_{F}=4$. By this point, the error in the one-way Euler solution is two orders of magnitude lower than the error in the PSE solution. The plateau in the one-way Euler convergence occurs when the discretization error becomes larger than the parabolization error.

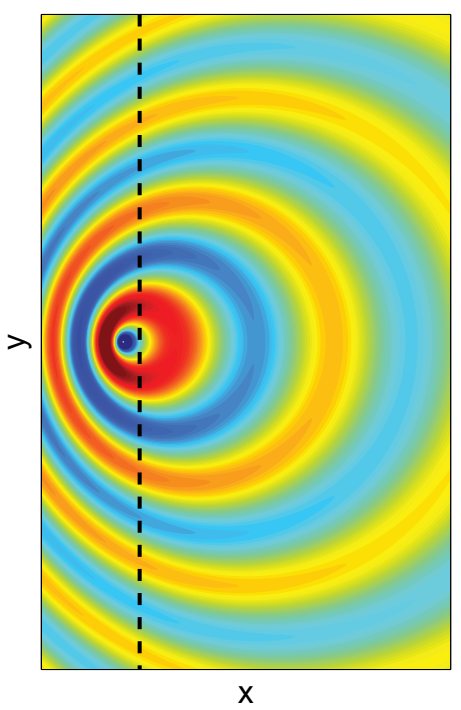

(a)

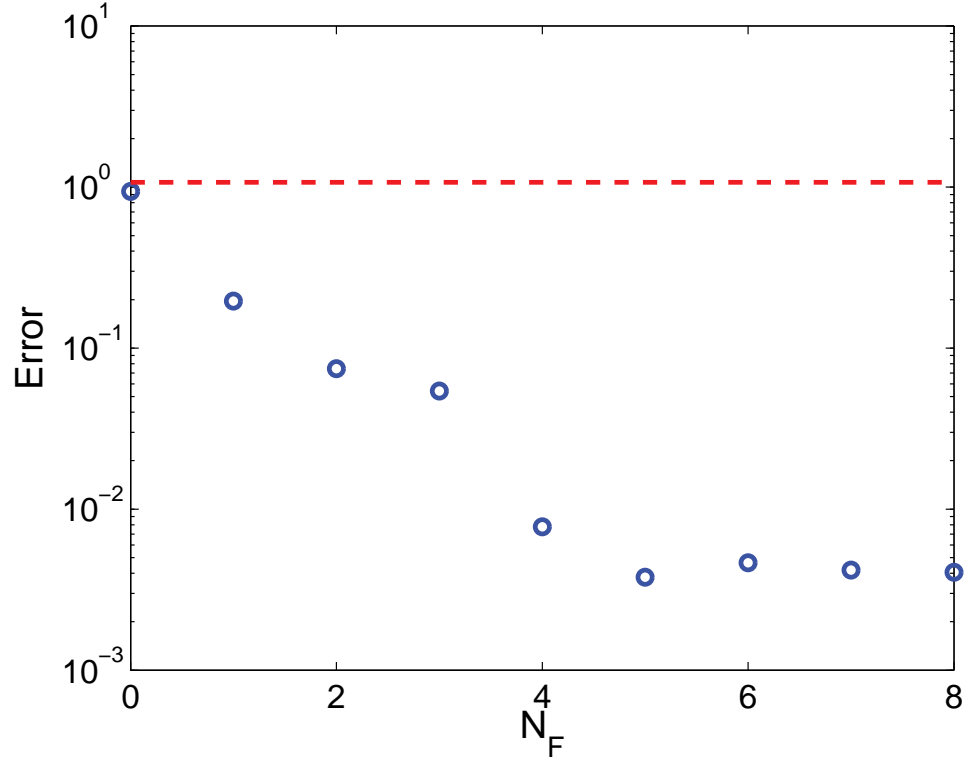

(b)

Figure 2: Monopole test case. (a) real part of the pressure field for the exact monopole solution. The dashed line shows the location where the initial condition is specified for the marching procedures. (b) global error of one-way Euler equations (symbols) and PSE (line) compared to the exact solution. Error is defined as $\left\|\left(\hat{p}-\hat{p}_{\text {exact }}\right) / \hat{p}_{\text {exact }}\right\|_{2}$ integrated over the full computational domain.

A useful way to visualize the convergence of the parabolization procedure is to compare the eigenvalues of the one-way Euler operator to those of the original Euler equations. Precisely, we compare the eigenvalues of $P$ (see equation (15)) against those of the one-way operator given in equation (20a) for different values of the order of the recursion. Figure 3 shows these eigenvalues for several of the operators used in the monopole example problem above. Note that additional stable convective modes exist as in figure 1, but here we have omitted these eigenvalues from the plot for readability. Also, the moderate change in shape of the acoustic branches compared to figure 1 is caused by the radiation boundary conditions, which are not enforced for the idealized plane waves used to derive the analytical modes.

Recall that the goal of our method is to generate an operator that accurately contains the downstream Euler modes but does not include any upstream acoustic modes. That this goal is achieved is clearly demonstrated by figure 3 . The $N_{F}=0$ case corresponds to performing no filtering and depending entirely upon the partial decoupling of the dynamics realized by the transformation to one-dimensional characteristic variables . Even with no filtering, the upstream acoustic waves have been removed. The very small angle downstream propagating waves are properly captured while all other waves are poorly modeled. This is consistent with our previous discussion about the nature of the one-dimensional characteristic variables.

As $N_{F}$ is increased, more and more of the downstream acoustic modes are properly modeled. At $N_{F}=4$, all propagating waves are well resolved. Increasing $N_{F}$ beyond this point primarily effects less important evanescent modes, although close examination revels that all modes continue to converge toward the spectrum of the original discretized Euler equations. Note that convergence to the spectrum of $P$ implies that we have represented all waves as well as possible for the given transverse discretization. The properties of the underlying discretization are unaltered by the parabolization method. One manifestation of this is that the eigenvalues of the parabolized operator converge to the physical and unphysical modes that exist in the original discretized Euler equations. This behavior is evident in figure 3. 


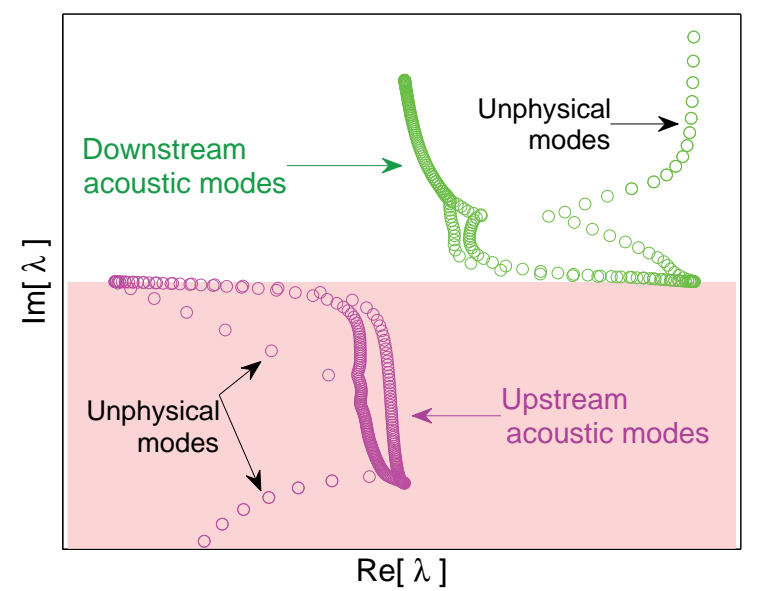

(a) Original Euler

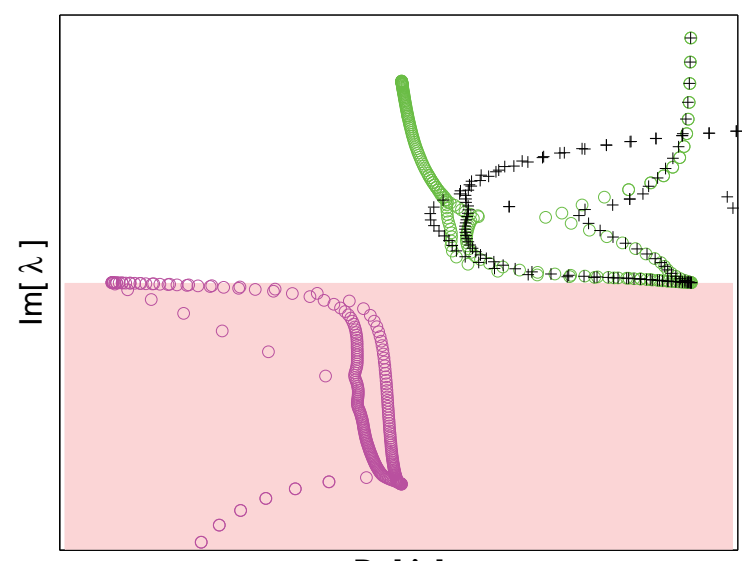

$\operatorname{Re}[\lambda]$

(c) $N_{F}=2$

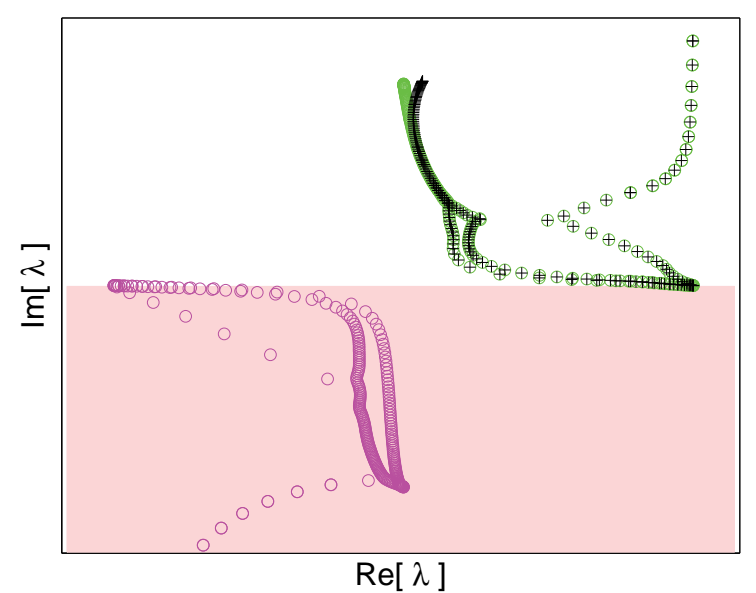

(e) $N_{F}=6$

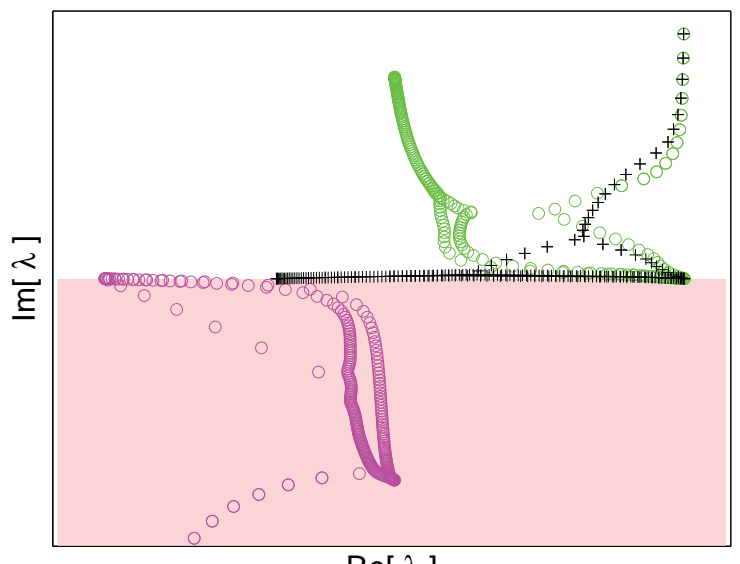

$\operatorname{Re}[\lambda]$

(b) $N_{F}=0$

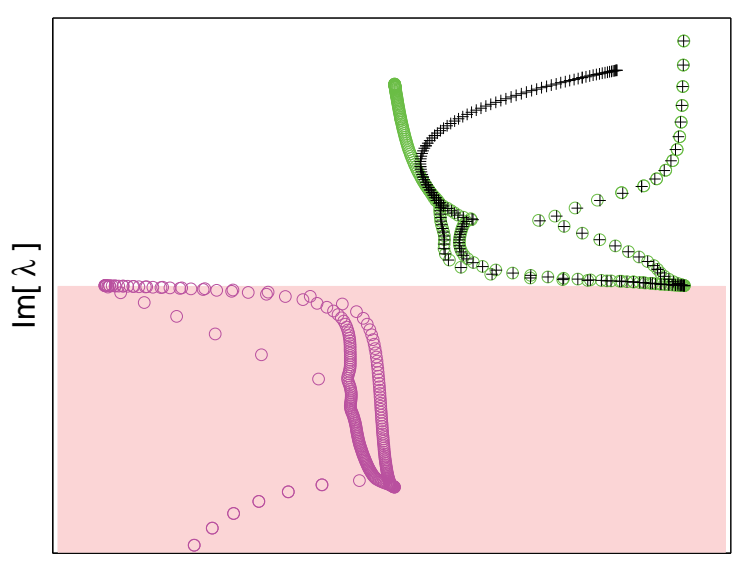

$\operatorname{Re}[\lambda]$

(d) $N_{F}=4$

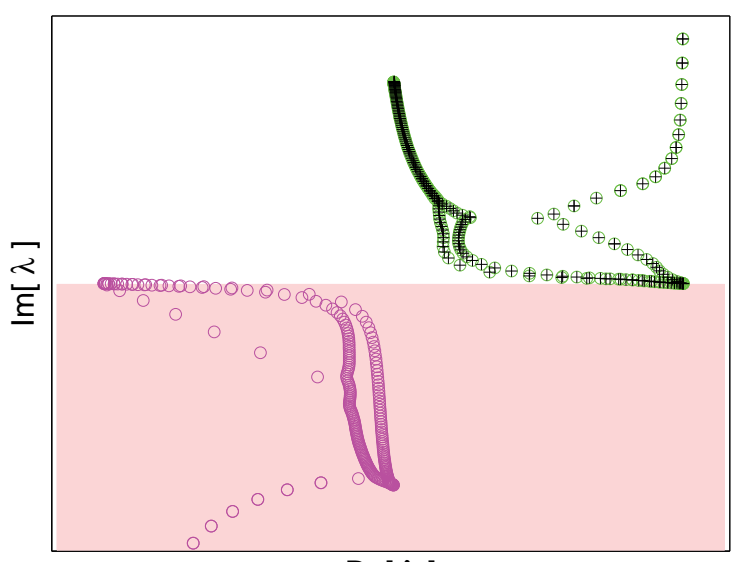

$\operatorname{Re}[\lambda]$

(f) $N_{F}=8$

Figure 3: Convergence of the spectrum of the one-way operator to the downstream modes of the original Euler operator. The upstream waves are completely removed and the downstream waves converge rapidly. The circles are the original Euler acoustic modes, and the pluses are the one-way Euler acoustic modes. 


\section{III.B. Turbulent mixing layer}

Here, we model the generation and propagation of sound in a turbulent mixing layer. The baseflow is a second-order approximation of a self-similar solution of the incompressible boundary layer equations using Prandtl's eddy viscosity model. ${ }^{27}$ The Mach number of the fast and slow free-streams are 0.8 and 0.2 , respectively. The shear layer thickness grows linearly, with the spread rate chosen to match experiments. The length scale is chosen such that the vorticity thickness is one at the inlet. We show results for the most unstable frequency at the inlet. The computational domain extends 150 vorticity thicknesses in the transverse direction and 100 vorticity thicknesses in the axial direction. We retain such a large domain in order to clearly show the far-field acoustics. This domain is discretized using 3000 points in $y$. The one-way Euler solution uses a step size of $\Delta x=0.05$ while the PSE solution uses $\Delta x=1$, which is just above the PSE minimum step size restriction for this problem. See the beginning of section III for additional computational details.

The initial condition at the inlet is a pure Kelvin-Helmholtz eigenfunction computed at $x=0$ under a locally-parallel assumption. Since the initial condition does not include any acoustic disturbances, all sound in the flow is created by the growth, decay, and distortion of the Kelvin-Helmholtz mode. Because of the central role of this mode, it is critical that the one-way Euler operator includes it accurately. Figure 4 shows that the eigenvalue of the Kelvin-Helmholtz mode in the one-way Euler operator converges spectrally to the eigenvalue of the Kelvin-Helmholtz mode in the original Euler operator. The acoustic modes converge in a similar manner. For the upcoming results, we use $N_{F}=15$.

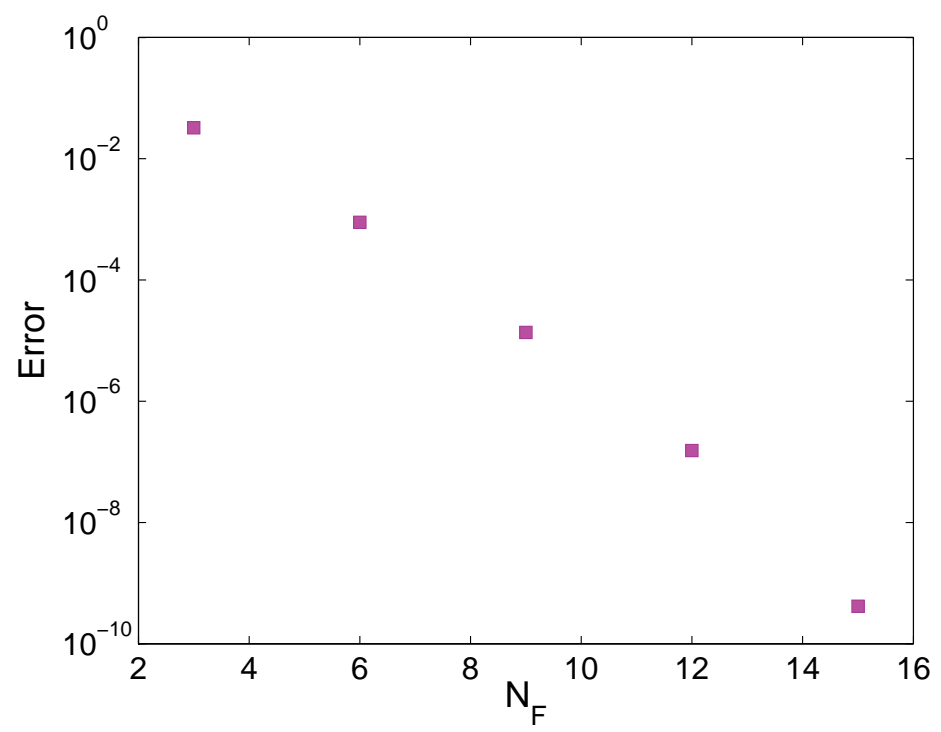

Figure 4: Convergence of the one-way Euler Kelvin-Helmholtz eigenvalue to the original Euler KelvinHelmholtz eigenvalue. Error is defined as the magnitude of the difference between the one-way Euler eigenvalue and the original Euler eigenvalue normalized by the magnitude of the original eigenvalue.

Figure 5 shows the pressure field as computed by the PSE and the one-way Euler equations. Comparing the two solutions reveals two benefits of the later method. First, notice the difference in the near-field wavepacket - the PSE wavepacket decays faster than the one-way Euler wavepacket. These near-field dynamics are dominated by the growth and decay of the Kelvin-Helmholtz mode. We have shown in figure 4 that the Kelvin-Helmholtz mode in the one-way operator is converged to the correct value. At $N_{F}=15$, the error is less than $10^{-9}$, so the accumulated parabolization error over the length of the domain is less than $\exp \left(100 \times 10^{-9}\right) \approx 10^{-7}$. The error due to the discretization is also small because of the small step size and fourth-order integration.

The overly rapid decay of the near-field wavepacket in the PSE solution can be explained by the implicit Euler integration of the PSE equations. In this flow, the Kelvin-Helmholtz mode is most unstable very near the inlet, so the growth rate is decreasing (or the decay rate is increasing) over the majority of the domain. Therefore, over each step in the PSE march, the growth rate is lower (or the decay rate is higher) at the downstream side of the interval than at the upstream side. Because of the implicit Euler integration, the 


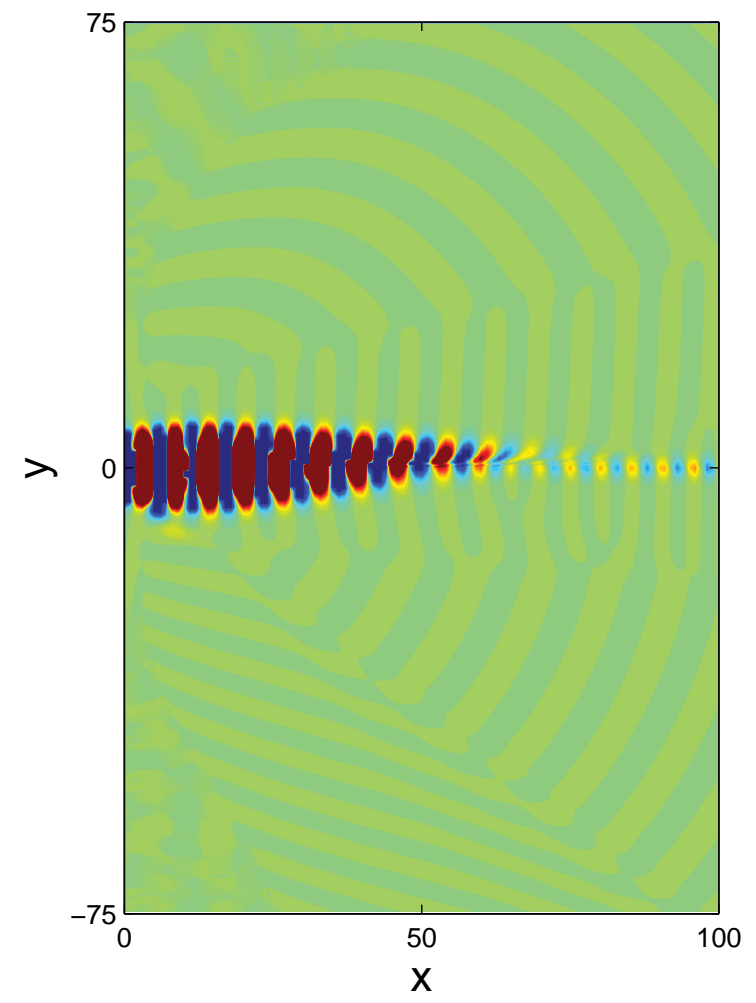

(a)

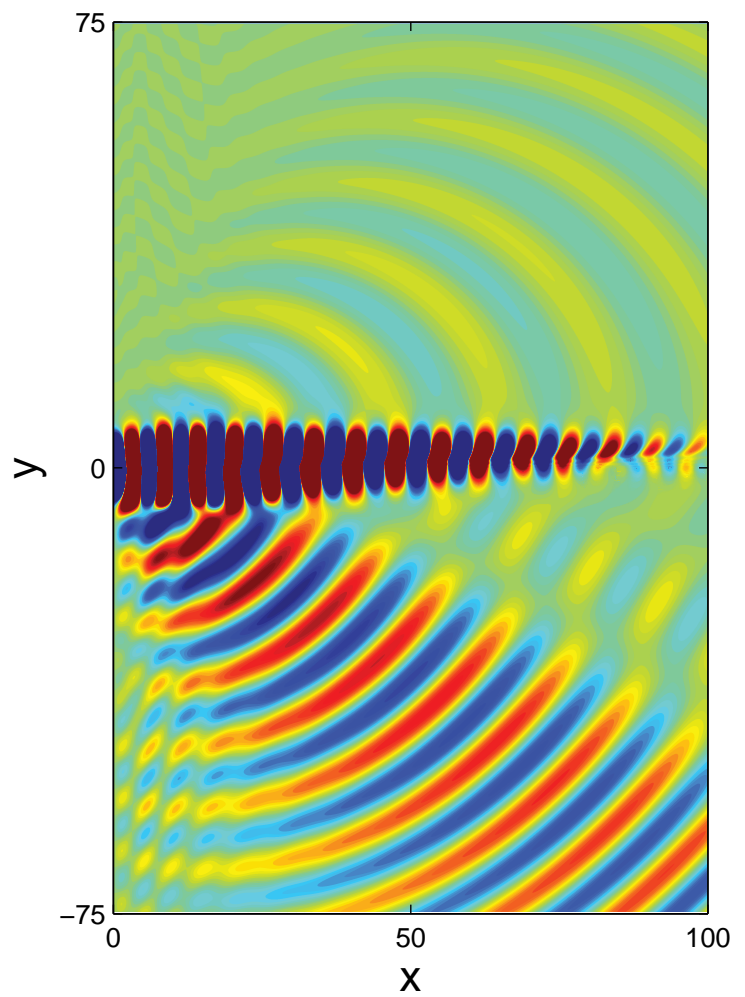

(b)

Figure 5: Pressure field from the turbulent mixing layer test case for (a) PSE and (b) the one-way Euler equation.

downstream value is applied over the entire interval, so the evolution of the mode is always biased toward a more damped solution. As the step size decreases, the integration converges toward the correct result, but satisfactory convergence cannot be achieved prior to the violation of the minimum step size restriction required for stability of the PSE march.

Second, the one-way Euler solution contains a well-resolved acoustic field while the PSE solution contains almost no acoustic field at all. The downstream acoustic modes in the PSE equations were heavily damped in order to adequately damp the upstream acoustic modes to allow a stable march. The downstream acoustic modes in the one-way Euler operator, on the other hand, were properly retained, allowing the strong downstream radiation produced by the peaking and subsequent decay of the Kelvin-Helmholtz mode to be captured.

In figure 6(b), we show a slice of the pressure field along $x=25$. In the near-field, the PSE and one-way Euler results are similar, with the minor discrepancy caused by the growth rate error discussed above. The exponential decay of the near-field pressure is consistent with the asymptotic behavior of the Kelvin-Helmholtz mode. The one-way Euler pressure field levels off due to the presence of propagating (non-decaying) acoustic waves that radiate to the far-field. In contrast, the damped remnants of acoustic radiation in the PSE solution is at least four order of magnitude too small. These results are consistent with comparisons made between DNS and PSE by Cheung \& Lele ${ }^{28}$ for laminar subsonic mixing layers.

Lastly, we address the irregular waveforms visible in the one-way Euler solution at high very high angles near $x=0$. This error is due to slow convergence of a hand-full of high angle acoustic modes. These modes do converge as the order of the filtering is increased, but more slowly than the other modes in the Euler spectrum. Therefore, this error can be removed, but the filtering order required to do so is much higher than necessary for all other modes. Eliminating this non-uniform convergence is one of the goals of our ongoing efforts to derive optimal filtering parameters. 


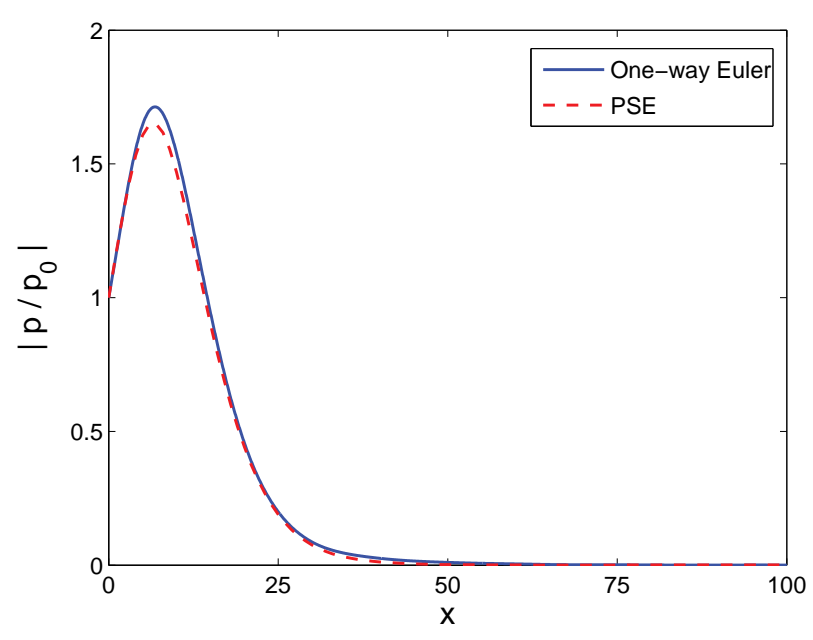

(a)

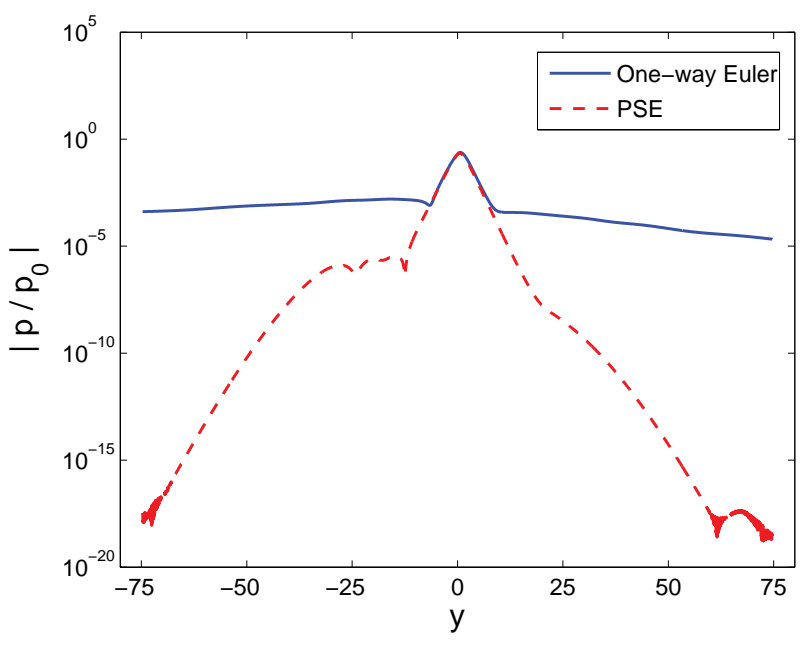

(b)

Figure 6: One-way Euler and PSE pressure amplitude for the turbulent mixing layer (a) along the axial slice $y=0$ (b) along the transverse slice $x=25$.

\section{Summary}

We have developed a one-way Euler equation that can be efficiently and stably solved as a spatial initial value problem. The one-way operator contains no upstream propagating modes, and can represent all downstream modes with arbitrary accuracy.

For problems where the mid- and far-field acoustic radiation is of interest, this technique will provide significant improvements over PSE. For example, we plan to use this technique as a reduced order model to obtain efficient jet noise predictions.

\section{Acknowledgments}

The authors gratefully acknowledge support from the Office of Naval Research under contract N001411-1-0753 with Dr. Brenda Henderson as technical monitor, and from NAVAIR under STTR contract N68335-11-C-0026 with Dr. John Spyropoulos as technical monitor. Additionally, the authors would like to thank Professor Thomas Hagstrom, Southern Methodist University and Professor Peter Schmid, École Polytechnique, for their helpful input on this work.

\section{References}

${ }^{1}$ Crow, S. and Champagne, F., "Orderly structures in jet turbulence," J. FLuid Mech., Vol. 48, 1971, pp. 547-591.

${ }^{2}$ Hussain, F., "Coherent structures and turbulence," J. Fluid Mech., Vol. 173, 1986, pp. 303-356.

${ }^{3}$ Viswanathan, K., "Aeroacoustics of hot jets," J. Fluid Mech., Vol. 516, 2004, pp. 39-82.

${ }^{4}$ Hileman, J., Thurow, B., Caraballo, E., and Samimy, M., "Large-scale structures evolution and sound emission in highspeed jets: real-time visualization with simultaneous acoustic measurements," J. Fluid Mech., Vol. 544, 2005, pp. 277-307.

${ }^{5}$ Zaman, K. B. M. Q. and Hussain, A. K. M. F., "Vortex pairing in a circular jet under controlled excitation. Part 1. General jet response," Journal of Fluid Mechanics, Vol. 101, 1980, pp. 449-491.

${ }^{6}$ Suzuki, T. and T.Colonius, "Instability waves in a subsonic round jet detected using a near-field phased microphone array," J. Fluid Mech., Vol. 565, 2006, pp. 197-226.

${ }^{7}$ Michalke, A., "Survey on jet instability theory," Progress in Aerospace Sciences, Vol. 21, No. 0, 1984, pp. 159 - 199.

${ }^{8}$ Tam, C. K. W. and Burton, D. E., "Sound generated by instability waves of supersonic flows. Part 2. Axisymmetric jets," J. Fluid Mech., Vol. 138, 1984, pp. 273-295.

${ }^{9}$ Nichols, J. and Lele, S., "Global modes and transient response of a cold supersonic jet," J. Fluid Mech., Vol. 669, 2011, pp. 225-241.

${ }^{10}$ Herbert, T., "Parabolized stability equations," Annu. Rev. Fluid Mech., Vol. 29, 1997, pp. 245-283.

${ }^{11}$ Gudmundsson, K. and Colonius, T., "Instability wave models for the near-filed fluctuations of turbulent jets," J. Fluid Mech., Vol. 689, 2011, pp. 97-128. 
${ }^{12}$ Colonius, T., Samanta, A., and Gudmundsson, K., "Parabolized stability equation models of large-scale jet mixing noise," IUTAM Symposium on Computational Aero-Acoustics for Aircraft Noise Prediction, Southampton, UK, March, 2010.

${ }^{13} \mathrm{Li}, \mathrm{F}$. and Malik, R., "Spectral analysis of the parabolized stability equations," Computers E Fluids, Vol. 26, No. 3, 1997, pp. 279-297. 273.

${ }^{14} \mathrm{Li}$, F. and Malik, M., "On the Nature of PSE Approximation," Theoret. Comput. Fluid Dynamics, Vol. 8, 1996, pp. 253-

${ }^{15}$ Andersson, P., Henningson, D., and Hanifi, A., "On a stabilization procedure for the parabolic stability equations," J. Engr. Mech., Vol. 33, 1998, pp. 311-332.

${ }^{16}$ Guddati, M., "Arbitrarily wide-angle wave equations for complex media," Comput. Methods Appl. Mech. Engrg., Vol. 195, 2006, pp. 65-93.

${ }^{17}$ Hagstrom, T., Watburton, T., and Givoli, D., "Radiation boundary conditions for time-dependent waves based on complete plane wave expansions," J. Comput. App. Math., Vol. 45, No. 4, 2007, pp. 1988-1995.

${ }^{18}$ Halpern, L. and Trefethen, L., "Wide-angle one-way wave equations," J. Acoust. Soc. Am., Vol. 4, 1988, pp. 890-901.

${ }^{19}$ Ehrhardt, M. and Zisowsky, A., "Discrete non-local boundary conditions for split-step Pade approximations of the one-way Helmholtz equation," J. of Comp.and Applied Math., Vol. 200, 2007, pp. 471-490.

${ }^{20}$ Givoli, D. and Neta, B., "High-order nonreflecting boundary scheme for time- dependent waves," J. Comput. Phys., Vol. 186, 2003, pp. 24-46.

${ }^{21}$ Hagstrom, T. and Warburton, T., "A new auxiliary variable formulation of high-order local radiation boundary conditions: corner compatibility conditions and extensions to first-order systems," Wave Motion, Vol. 39, 2004, pp. 890-901.

${ }^{22}$ Hagstrom, T., Topics in Computational Wave Propagation, vol.31 of Lecture Notes in Computational Science and Engineering, Vol. 70, chap. New results on absorbing layers and radiation boundary conditions, Springer-Verlag, New York, 2003, pp. 1-42.

${ }^{23}$ Hagstrom, T., Mar-Or, A., and Givoli, D., "High-order local absorbing conditions for the wave equation: extensions and improvements," AJ. of Comput. Phys., Vol. 227, 2008, pp. 3322-3357.

${ }^{24}$ Becache, E., Givoli, D., and Hagstrom, T., "High-order absorbing boundary conditions for anisotropic and convective wave equations," J. Comput. Phys., Vol. 229, 2010, pp. 1099-1129.

${ }^{25}$ Colonius, T. and Ran, H., "A super-grid-scale model for simulating compressible flow on unbounded domains," J. Comput. Phys., Vol. 182, No. 1, 2002, pp. 191-212.

${ }^{26}$ Appelo, D. and Colonius, T., "A high-order super-grid-scale absorbing layer and its application to linear hyperbolic systems," J. Comput. Phys., Vol. 228, No. 11, 2009, pp. 4200-4217.

${ }^{27}$ Yule, A., "Two-Dimensional Self-Preserving Turbulent Mixing Layers at Different Free Stream Velocity Ratios," Tech. rep., University of Manchester, 1972.

${ }^{28}$ Cheung, L. and of Mechanical Engineering, S. U. D., Aeroacoustic Noise Prediction and the Dynamics of Shear Layers and Jets Using the Nonlinear Parabolized Stability Equations, Stanford University, 2007. 